\title{
Optical pumping in a microfabricated Rb vapor cell using a microfabricated Rb discharge light source
}

\author{
V. Venkatraman, ${ }^{1, \text { a) }}$ S. Kang, ${ }^{1, b)}$ C. Affolderbach, ${ }^{1}$ H. Shea ${ }^{2}$ and G. Mileti ${ }^{1, c)}$ \\ ${ }^{1}$ Laboratoire Temps-Fréquence, University of Neuchâtel, Neuchâtel 2000, Switzerland \\ ${ }^{2}$ Microsystems for Space Technologies Laboratory, Ecole Polytechnique Fédérale de Lausanne (EPFL), \\ Neuchâtel 2002, Switzerland
}

(Received 27 December 2013; accepted 21 January 2014; published online 4 February 2014)

\begin{abstract}
Miniature $\left(<\right.$ few $\left.\mathrm{cm}^{3}\right)$ vapor-cell based devices using optical pumping of alkali atoms, such as atomic clocks and magnetometers, today mostly employ vertical-cavity surface-emitting lasers as pump light sources. Here, we report on the demonstration of optical pumping in a microfabricated alkali vapor resonance cell using (1) a microfabricated $\mathrm{Rb}$ discharge lamp light source, as well as (2) a conventional glass-blown $\mathrm{Rb}$ discharge lamp. The microfabricated $\mathrm{Rb}$ lamp cell is a dielectric barrier discharge (DBD) light source, having the same inner cell volume of around $40 \mathrm{~mm}^{3}$ as that of the resonance cell, both filled with suitable buffer gases. A miniature $\left(\sim 2 \mathrm{~cm}^{3}\right.$ volume) test setup based on the $\mathrm{M}_{\mathrm{z}}$ magnetometer interrogation technique was used for observation of optical-radiofrequency double-resonance signals, proving the suitability of the microfabricated discharge lamp to introduce efficient optical pumping. The pumping ability of this light source was found to be comparable to or even better than that of a conventional glass-blown lamp. The reported results indicate that the micro-fabricated DBD discharge lamp has a high potential for the development of a new class of miniature atomic clocks, magnetometers, and quantum sensors. (C) 2014 AIP Publishing LLC. [http://dx.doi.org/10.1063/1.4864080]
\end{abstract}

Optical pumping ${ }^{1}$ of alkali atoms in vapor-cells has been extensively used for decades to create several high precision devices such as compact $\left(100-1000 \mathrm{~cm}^{3}\right)$ atomic clocks, ${ }^{2,3}$ magnetometers, ${ }^{4,5}$ gyroscopes, ${ }^{6}$ and several others. ${ }^{7-9}$ With the advent of microtechnology and the rising number of portable applications ${ }^{10-12}$ (global positional system receivers, remote sensing, and magnetocardiography), there has been considerable interest in miniaturizing these device ${ }^{13-15}$ to the few $\mathrm{mm}^{3}$-few $\mathrm{cm}^{3}$ volume range while compromising only a little in its performance. A pumping light source is required in all the alkali vapor cell devices to sustain a population imbalance between certain atomic levels. Commercial alkali ( $\mathrm{Rb}$, $\mathrm{K}$, or $\mathrm{Cs}$ ) atomic clocks and magnetometers have mostly used discharge lamps ${ }^{16,17}$ for this purpose as they intrinsically emit the necessary spectral lines for optical pumping of the used atomic species, with very low frequency drifts. ${ }^{18}$ Most of the recent miniature demonstrators and devices however typically use a laser diode (VCSEL_-Vertical Cavity Surface Emitting Laser) as the pumping light source to emit the required alkali D-lines. ${ }^{10,19}$ This was mainly because the inductively coupled glass-blown (GB) alkali discharge lamps, with their hardto-integrate cylindrical/spherical geometry and high power consumption (several Watts), could not be scaled down to meet the size and power requirements of miniature clocks, and because the Coherent Population Trapping (CPT) scheme often employed for miniature atomic clocks requires two phase-coherent light fields that is usually provided by a laser

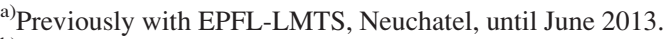

${ }^{b)}$ Permanent address: Key Laboratory of Atomic Frequency Standards, Chinese Academy of Sciences, Wuhan Institute of Physics and Mathematics, Wuhan 430071, China.

${ }^{c)}$ Author to whom correspondence should be addressed. Electronic mail: gaetano.mileti@unine.ch
}

source. ${ }^{20}$ VCSELs are compact, energy efficient, and operate at low power, and hence were suitable choices. ${ }^{21}$ However, they have several undesirable characteristics including a strong temperature dependence of the output wavelength, ageing effects, and higher light output noise levels than discharge lamps. ${ }^{22}$ Alkali discharge lamps, on the other hand, do not require any sophisticated control electronics (necessary for a laser diode) to stabilize their emission wavelength, as the wavelength has very low temperature dependence and ageing, and is intrinsically at the right atomic resonance wavelength.

Only very few studies towards the use of miniaturized discharge lamps in atomic devices have been reported ${ }^{23,24}$ previously. Recently, our teams have developed a miniature microfabricated $1 \times 1 \times 0.3 \mathrm{~cm}^{3} \mathrm{Rb}$ dielectric barrier discharge (DBD) light source ${ }^{25}$ as an alternative to a VCSEL light source and to extend the inherent advantages of $\mathrm{a} \mathrm{Rb}$ discharge lamp to miniature clocks and magnetometers. This light source was also characterized with respect to various cell and drive parameters to minimize its power consumption and increase D-line emission. ${ }^{26}$ In this present article, we report on the optical pumping ability of the Rb DBD light source (Fig. 1(b)), tested and observed using a miniature $\mathrm{M}_{\mathrm{z}}$ magnetometer experimental setup with a microfabricated $\mathrm{Rb}$ resonance cell (Fig. 1(a)).

The $M_{z}$ magnetometer interrogation technique ${ }^{27}$ was chosen for the demonstration of Zeeman optical pumping, as it is straightforward to implement (without the need of a microwave resonator or $\mathrm{Rb}$ isotopic filters for hyperfine optical pumping). In this technique, circularly polarized $(\sigma+$, for instance) $\mathrm{Rb} \mathrm{D} 1 / \mathrm{D} 2$ light is made to propagate in the direction of the wave vector $(\vec{k})$ through a sample of $\mathrm{Rb}$ atoms, parallel to the ambient DC magnetic field $\left(\vec{B}_{d c}\right)$ as shown 


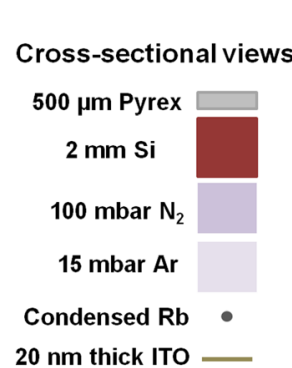

FIG. 1. Photographs and cross-sectional schematic views of the (a) microfabricated $\mathrm{Rb}$ resonance cell and (b) the Rb DBD light source.

schematically in Fig. 2(a). The Rb hyperfine levels are further split into Zeeman sub-levels due to presence of $\vec{B}_{d c}$, as shown schematically in Fig. 2(b). The $\sigma+$ light pumps the $\mathrm{Rb}$ atoms from the $5 \mathrm{~S}_{1 / 2} \mathrm{~m}_{\mathrm{F}}$ to $5 \mathrm{P}_{1 / 2} \mathrm{~m}_{\mathrm{F}+1}$ levels because the circular polarization adds angular momentum to the excitations. Eventually, the $5 \mathrm{~S}_{1 / 2}$ high- $\mathrm{m}_{\mathrm{F}}$ state $\left(\mathrm{F}=2 \mathrm{~m}_{\mathrm{F}}=+2\right)$ becomes strongly populated resulting in a population imbalance between the $m_{F}$ sublevels. The $\mathrm{Rb}$ atoms are also exposed to a radio-frequency (RF) magnetic field $\left(\vec{B}_{r f}\right)$, oriented perpendicular to the $\vec{B}_{d c}$ direction. If the RF field is in resonance with the Zeeman splitting, transitions between the $5 \mathrm{~S}_{1 / 2} \mathrm{~m}_{\mathrm{F}}$ and $5 \mathrm{~S}_{1 / 2} \quad \mathrm{~m}_{\mathrm{F}-1}$ levels are induced (for $\sigma+$ light). This leads to an increased absorption of the D light, which is detected using a photo diode. This signal that arises as a result of the interrogating optical and radio-frequency fields is called the Double-Resonance (DR) signal. The resonance frequency or Larmor frequency, $\mathrm{f}_{\mathrm{L}}$, corresponding to the Zeeman splitting, can be defined by the equation: $B_{d c}=f_{L} / \gamma$, where $\gamma$ is termed the gyromagnetic ratio. $\gamma_{85}=4.66 \mathrm{kHz} / \mu \mathrm{T}$ for ${ }^{85} \mathrm{Rb}$ and $\gamma_{87}=7 \mathrm{kHz} / \mu \mathrm{T}$ for ${ }^{87} \mathrm{Rb}$.
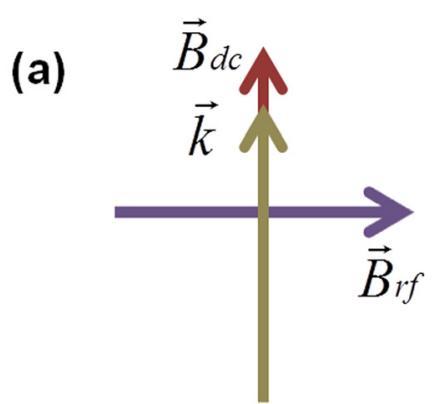

(b)

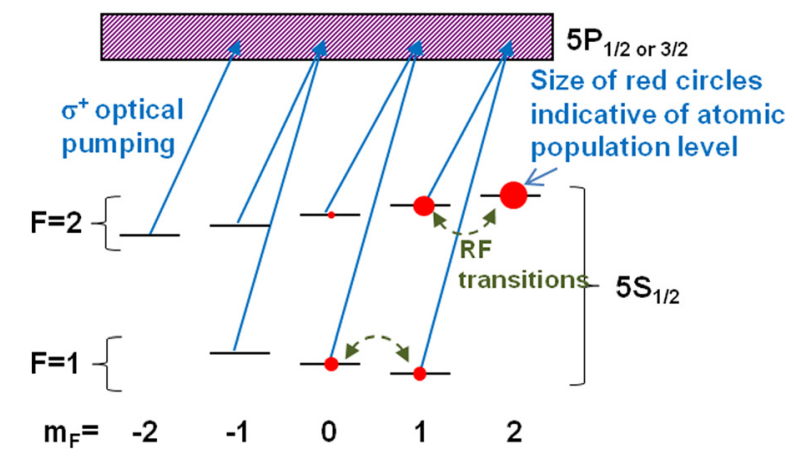

FIG. 2. Schematic representations of the (a) light field and magnetic field vectors for $M_{z}$ interrogation and (b) energy level scheme of the Rb ground states showing the optical and RF double-resonance transitions. (a)
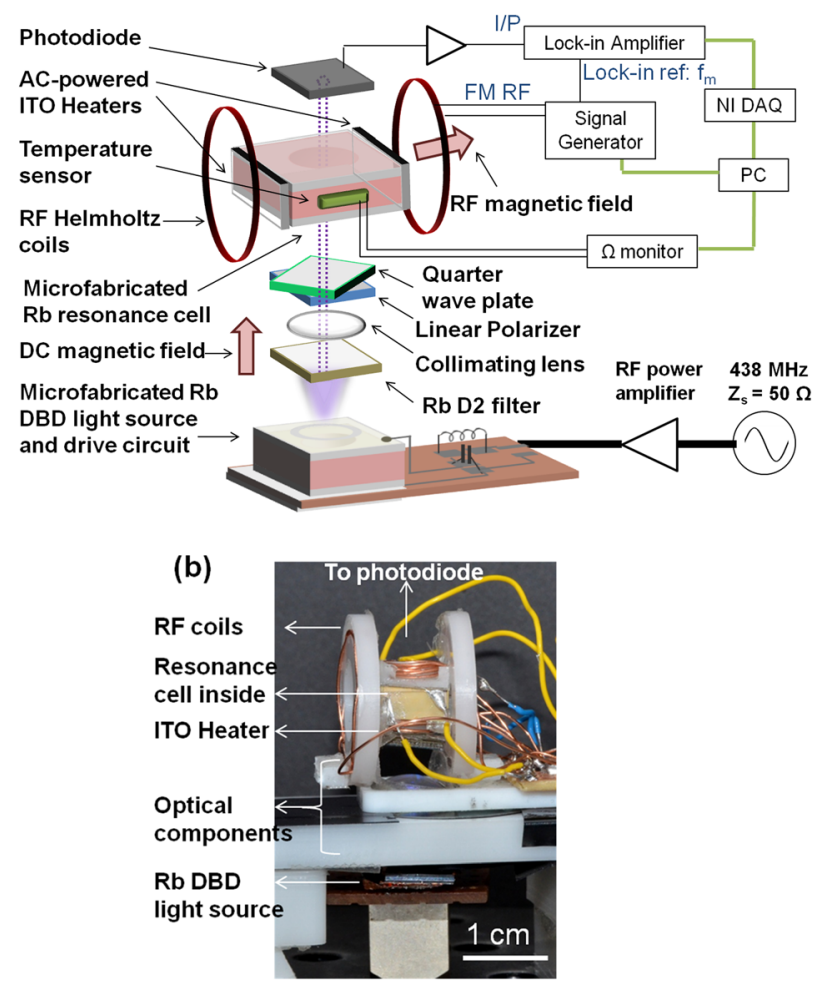

FIG. 3. Schematic of the miniature $\mathrm{M}_{\mathrm{z}}$ magnetometer test setup for the optical pumping demonstration.

The experimental setup is a vertical stack configuration with the light source placed on the bottom and emitting light upwards, as shown schematically in Fig. 3(a). A side-view photograph of the setup is also shown in Fig. 3(b). The Rb DBD light source, with ${ }^{87} \mathrm{Rb}$ and 15 millibars Ar, had a $20 \mathrm{~nm}$ thick ITO (Indium Tin Oxide) thin film layer as the top electrode and a $200 \mathrm{~nm}$ thick Al layer as the bottom electrode (see Fig. 1). The light source was impedance matched to 50 $\Omega$ and driven at $438 \mathrm{MHz}$. A band-pass filter tuned to the $\mathrm{Rb}$ D2 line (Center wavelength: $780 \mathrm{~nm} \pm 10 \mathrm{~nm}$, Edmund Optics 65-178) was placed just above the light source, and an aspheric collimating lens was used to maximize the light through the resonance cell. A D2 filter was used because the D2 line was observed to be $20 \%$ more efficient in pumping when compared to D1 [A DR signal can also be obtained without using any D-line filters]. The output D2 line intensity from the lamp was also found to be twice that of D1 for a given drive input power to the lamp. A linear polarizer (LP) and a quarter wave plate (both $1 \mathrm{~mm}$ thick) were aligned to produce the circularly polarized light needed for the Zeeman pumping.

The resonance cell (Fig. 1(a)) is a microfabricated $\mathrm{Rb}$ cell (cylindrical inner volume: $5 \mathrm{~mm}$ height and $2.5 \mathrm{~mm}$ radius) with natural $\mathrm{Rb}$ isotopic mixture and 100 millibars of $\mathrm{N}_{2}$ as buffer gas. ${ }^{28}$ A high buffer gas pressure was chosen to reduce the number of depolarizing $\mathrm{Rb}$ atomic collisions with the cell walls and hence increase pumping efficiency. ${ }^{13}$ Two $10 \times 3 \times 1 \mathrm{~mm}^{3}$ Pyrex substrates with a $20 \mathrm{~nm}$ thick ITO thin film were glued to opposing cell sidewalls and used to heat the resonance cell by connecting them to an AC power supply (1.1 kHz frequency used). A Pt1000 temperature sensor was glued on the third cell sidewall for measuring and controlling the resonance cell temperature. Two RF 


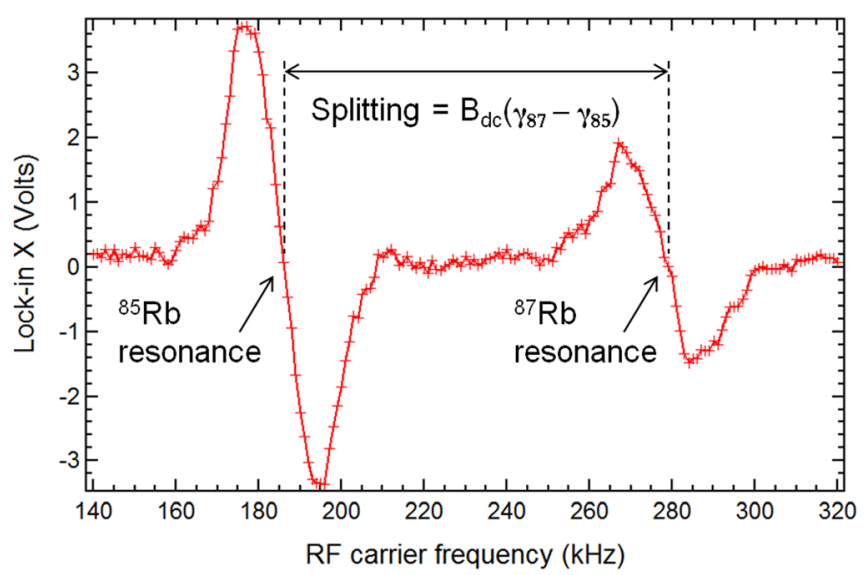

FIG. 4. Optical-RF DR signals at the respective ${ }^{85} \mathrm{Rb}$ and ${ }^{87} \mathrm{Rb}$ Larmor frequencies (corresponding to the ambient DC magnetic field strength of $40 \mu \mathrm{T})$ observed via the lock-in amplifier.

Helmholtz coils, 15 turns and $2 \mathrm{~cm}$ diameter, were placed to produce the homogeneous RF field across the cell volume, with the field vector perpendicular to the light propagation vector.

The photodiode ( $\mathrm{Si}$ broadband) current was amplified with a $10^{5} \mathrm{~V} / \mathrm{A}$ conversion coefficient, and this output voltage was given as input to a lock-in amplifier. Fig. 4 shows the DR signals of ${ }^{85} \mathrm{Rb}$ and ${ }^{87} \mathrm{Rb}$ observed from the lock-in for the ambient DC field of $40 \mu \mathrm{T}$. The RF field was frequency modulated at $\mathrm{f}_{\mathrm{m}}=414 \mathrm{~Hz}$ ( set as the lock-in reference frequency) with a frequency deviation, $\mathrm{f}_{\mathrm{dev}}=40 \mathrm{kHz}$, and field amplitude, $\mathrm{B}_{\mathrm{rf}}$, estimated to be around $8 \mu \mathrm{T}$. The carrier frequency, $\mathrm{f}_{\mathrm{c}}$, was swept around the expected resonance frequency range to observe the DR signal at the resonance frequency, $\mathrm{f}_{\mathrm{L}}$. The lock-in time constant was $300 \mathrm{~ms}$ and sensitivity was $20 \mu \mathrm{V}$. The resonance cell was temperature controlled at $74{ }^{\circ} \mathrm{C}$ as this temperature yielded the highest DR signal amplitude. $38 \mu \mathrm{W}$ of D2 light was incident on the resonance cell and $6 \mu \mathrm{W}$ reached the photodiode. Both DR signals were observed as expected for a natural isotopic mixture $\left(72 \%{ }^{85} \mathrm{Rb}\right.$ and $\left.28 \%{ }^{87} \mathrm{Rb}\right)$ containing $\mathrm{Rb}$ resonance cell. Their splitting and the ratio of their resonant frequencies also matched the ratio of their gyromagnetic ratios $\left(\gamma_{87} / \gamma_{85}\right)$. The power stability of the light emitted by the microfabricated discharge light source is on the level of few percent over the timescale of few minutes to few hours ${ }^{25,29}$ resulting in a reproducibility of the DR signals on the level of the noise in Fig. 4 between consecutive scans.

Optimum FM-RF interrogation parameters are necessary to achieve the desired performance of a magnetometer for the lowest $\mathrm{Rb} \mathrm{D}$ light input. This helps in minimizing the power consumption of the light source (and hence also avoids optical power broadening of the D lines) which is a critical factor in portable applications. Fig. 5 shows how the ${ }^{85} \mathrm{Rb}$ DR signal varies with change in the RF field $\mathrm{f}_{\mathrm{m}}$ and $f_{\text {dev }}$. The normalized signal slope (at the point of ${ }^{85} \mathrm{Rb}$ zero-crossing shown in Fig. 4) is the ratio of the DR signal amplitude to its line-width at full maximum. $\mathrm{f}_{\mathrm{m}}$ values less than around $500 \mathrm{~Hz}$ and $\mathrm{a} \mathrm{fev}_{\mathrm{dev}}$ value of around $40 \mathrm{kHz}$ were found to be optimal for this light source. For $\mathrm{f}_{\mathrm{m}}$ approximately below $150 \mathrm{~Hz}$, even though the signal strength was the best, the detection noise was high due to $1 / \mathrm{f}$ noise and hence the $150-500 \mathrm{~Hz}$ range is preferable.

For a comparative analysis, the light source was replaced with a conventional GB inductively coupled Rb lamp, similar to the type used in commercial devices. The GB lamp had ${ }^{87} \mathrm{Rb}$ with 2 millibars Ar and was cylindrical with $1 \mathrm{~cm}$ height and diameter. The same experimental conditions (used to observe the result shown in Fig. 4) were used for both the lamps and the observed ${ }^{85} \mathrm{Rb} \mathrm{DR}$-interrogation results are shown in Fig. 6. It can be seen that the signal amplitude achieved with the DBD lamp is only $\sim 10 \%$ lower than the GB lamp and the line-width is almost $50 \%$ lower than the GB lamp here. Overall, the DBD lamp provides a steeper signal slope than a conventional GB lamp at the center of resonance in this case.

The optical pumping ability of a miniature microfabricated $\mathrm{Rb}$ discharge light source has been demonstrated. Its pumping performance is comparable to or even better than a conventional glass-blown Rb lamp. Without any steps taken towards optimization, the current $\mathrm{M}_{\mathrm{z}}$ magnetometer sensitivity is $\sim 20 \mathrm{nT} / \sqrt{ } \mathrm{Hz}$. We expect that by (1) adding magnetic shields around the resonance cell, (2) reducing the field inhomogeneities introduced by the resonance cell heater, ${ }^{19}$ and (3) changing to the $M_{x}$ interrogation technique, ${ }^{30}$ the sensitivity can be greatly improved to create a high-performance
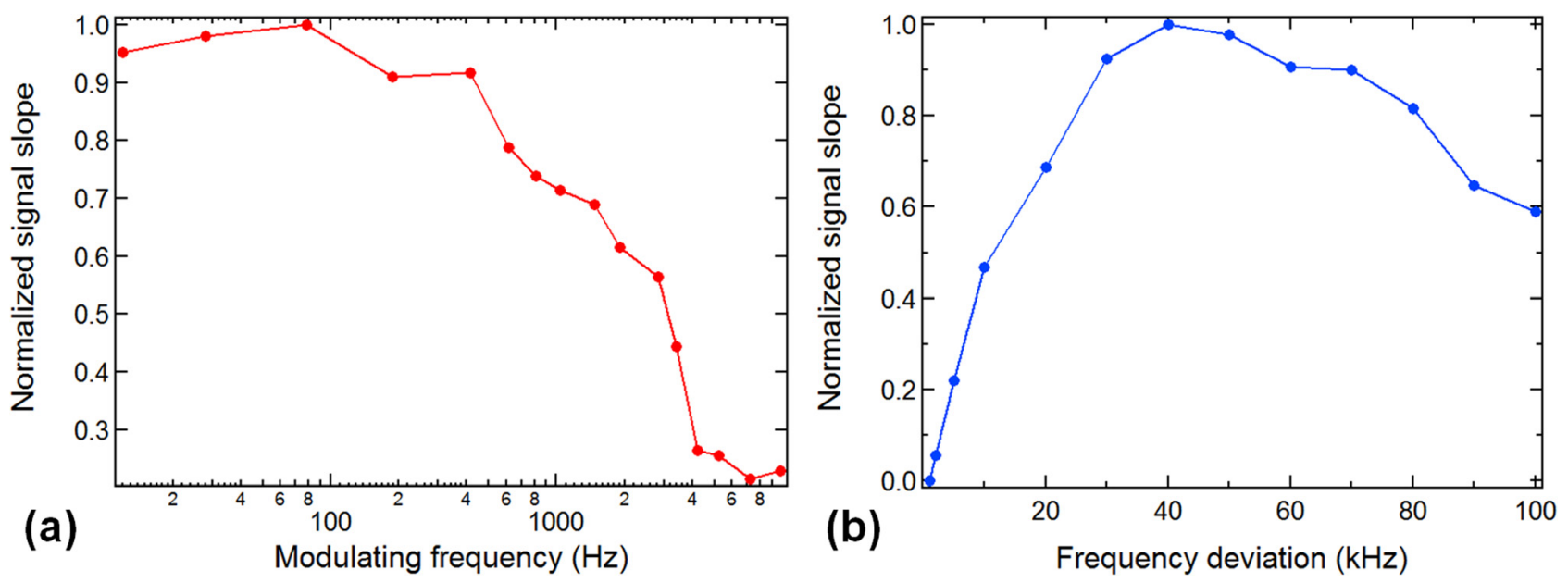

FIG. 5. The effect of the (a) RF field modulating frequency $\left(\mathrm{f}_{\mathrm{m}}\right)$ and (b) frequency deviation $\left(\mathrm{f}_{\mathrm{dev}}\right)$ values on the ${ }^{85} \mathrm{Rb} \mathrm{DR}$ signal detection. 


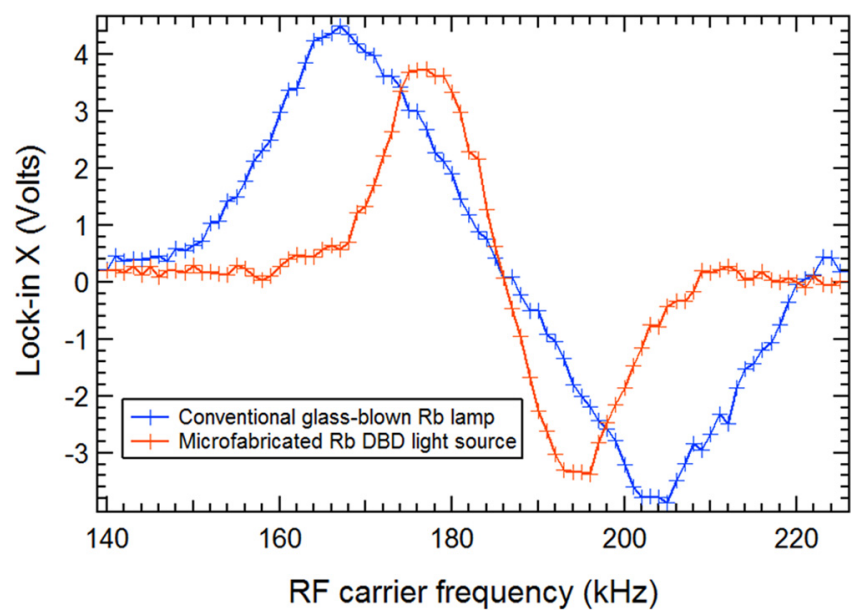

FIG. 6. Zoomed-in view of the ${ }^{85} \mathrm{Rb}$ DR signals (observed using a lock-in amplifier) when using different $\mathrm{Rb}$ lamps to compare their optical pumping abilities. Both the DR signals were found to be comparable and in the same order.

miniature DR magnetometer. It has been previously shown that using lamps or lasers as pumping light sources result in comparable magnetometer performances. ${ }^{31}$ For the envisaged application of the microfabricated lamp in a miniature DR atomic clock, next steps will require implementation of isotopic filtering ${ }^{24}$ for demonstration of hyperfine optical pumping $^{3}$ and combination with a miniaturized microwave cavity. 32,33

The DBD light sources can be batch-fabricated and can already be a good replacement for the currently commercially used glass-blown lamps in terms of size and power consumption. With an optimized magnetometer interrogation setup, only around $5 \mu \mathrm{W}$ of the D1/D2 line may be required from the light source for achieving a pT-level sensitivity. This means that the light source could be driven in the $10-15 \mathrm{MHz}$ range with a reduced power consumption. ${ }^{34}$ These results show promising potential for a new range of miniature DR-based atomic clocks, magnetometers, and other vapor-cell based devices.

The authors would like to acknowledge Swiss National Science Foundation (Sinergia Grant No. CRS120_122693) and Université de Neuchâtel for funding this research and Dr. Yves Pétremand (SAMLAB, EPFL) for providing the $\mathrm{Rb}$ vapor cells.

${ }^{1}$ W. Happer, Rev. Mod. Phys. 44, 169 (1972).

${ }^{2} \mathrm{~J}$. Vanier and C. Audoin, The Quantum Physics of Atomic Frequency Standards (2 volume set) (Adam Hilger, 1989).

${ }^{3}$ J. Camparo, Phys. Today 60(11), 33 (2007).

${ }^{4}$ D. Budker and M. Romalis, Nat. Phys. 3, 227 (2007).

${ }^{5}$ E. B. Alexandrov, M. V. Balabas, A. S. Pasgalev, A. K. Vershovskii, and N. N. Yakobson, Laser Phys. 6, 244 (1996).

${ }^{6}$ T. W. Kornack, R. K. Ghosh, and M. V. Romalis, Phys. Rev. Lett. 95, 230801 (2005).

${ }^{7}$ J. Fang and J. Qin, Sensors 12, 6331 (2012).
${ }^{8}$ A. Kuzmich, L. Mandel, and N. P. Bigelow, Phys. Rev. Lett. 85, 1594 (2000).

${ }^{9}$ M. Klein, I. Novikova, D. F. Phillips, and R. L. Walsworth, J. Mod. Opt. $\mathbf{5 3}, 2583$ (2006).

${ }^{10}$ S. Knappe, Compr. Microsyst. 3, 571 (2007).

${ }^{11}$ S. Mares, Introduction to Applied Geophysics (Springer, 1984).

${ }^{12}$ T. Sander, Biomed. Opt. Express 3, 981 (2012).

${ }^{13}$ J. Kitching, S. Knappe, and L. Hollberg, Appl. Phys. Lett. 81, 553 (2002).

${ }^{14}$ S. Knappe, T. H. Sander, O. Kosch, F. Wiekhorst, J. Kitching, and L. Trahms, Appl. Phys. Lett. 97, 133703 (2010).

${ }^{15}$ J. Kitching, S. Knappe, and E. A. Donley, IEEE Sens. J. 11, 1749 (2011).

${ }^{16}$ W. E. Bell, A. L. Bloom, and J. Lynch, Rev. Sci. Instrum. 32, 688 (1961).

${ }^{17}$ J. G. Coffer, M. Huang, and J. C. Camparo, "Self-pulsing in alkali rfdischarge lamps," in Proceedings of the IEEE International Frequency Control Symposium (FCS), Baltimore, U.S.A., 21-24 May, 2012, pp. 1-5.

${ }^{18}$ C. H. Volk, R. P. Frueholz, T. C. English, T. J. Lynch, and W. J. Riley, "Lifetime and reliability of rubidium discharge lamps for use," in 38th Annual Symposium on Frequency Control, Philadelphia, U.S.A., 29 May-1 June, 1984, pp. 387-400.

${ }^{19}$ P. Schwindt, S. Knappe, V. Shah, L. Hollberg, J. Kitching, L. Liew, and J. Moreland, Appl. Phys. Lett. 85, 6409 (2004).

${ }^{20}$ E. Arimondo, Prog. Opt. 35, 257 (1996).

${ }^{21}$ D. Serkland, G. Peake, K. Geib, R. Lutwak, R. Garvey, M. Varghese, and M. Mescher, "VCSELs for atomic clocks," Proc. SPIE 6132, 613208 (2006).

${ }^{22}$ R. Lutwak, D. Emmons, W. Riley, and R. Garvey, "The chip-scale atomic clock-coherent population trapping vs. conventional interrogation," in 34th Annual Precision Time and Time Interval Meeting, Reston, U.S.A., 2002, 3-5 December, pp. 539-550.

${ }^{23} \mathrm{H}$. Guo and A. Lal, "Self-powered photon source," in 12th International Conference in Solid State Sensors, Actuators, and Microsystems, Boston, U.S.A., 8-12 June, 2003, pp. 1474-1477.

${ }^{24}$ S. Wang, L. Lin, and H. Guo, "Analysis and design of a micromachined $\mathrm{Rb}-85$ filter in passive rubidium atomic clock," in 4th IEEE International Conference on Nano/Micro Engineered and Molecular Systems, Shenzhen, China, 5-8 January, 2009, pp. 911-914.

${ }^{25}$ V. Venkatraman, Y. Petremand, C. Affolderbach, G. Mileti, N. de Rooij, and H. Shea, IEEE Trans. Ultrason. Ferroelectr. Freq. Control 59, 448 (2012).

${ }^{26}$ V. Venkatraman, Y. Petremand, C. Affolderbach, G. Mileti, N. de Rooij, and H. Shea, "Optimization of a chip-scale Rb plasma discharge light source: Effects of RF drive frequency and cell impedance," in European Frequency and Time Forum (EFTF), Gothenburg, Sweden, 23-27 April, 2012.

${ }^{27}$ A. L. Bloom, Appl. Opt. 1, 61 (1962).

${ }^{28}$ Y. Petremand, C. Affolderbach, R. Straessle, M. Pellaton, D. Briand, G. Mileti, and N. F. De Rooij, J. Micromech. Microeng. 22, 025013 (2012).

${ }^{29}$ V. Venkatraman, F. Vecchio, T. Maeder, P. Ryser, and H. Shea, "LTCC integrated miniature $\mathrm{Rb}$ discharge lamp module for stable optical pumping in miniature atomic clocks and magnetometers," in 2012 IEEE 18th International Symposium for Design and Technology in Electronic Packaging (SIITME), Alba Iulia, Romania, 25-28 October, 2012, pp. 111-114.

${ }^{30}$ P. Schwindt, B. Lindseth, S. Knappe, V. Shah, J. Kitching, and L. A. Liew, Appl. Phys. Lett. 90, 081102 (2007).

${ }^{31}$ S. Groeger, A. S. Pazgalev, and A. Weis, Appl. Phys. B: Lasers Opt. 80, 645 (2005).

${ }^{32}$ A. M. Braun, T. J. Davis, M. H. Kwakernaak, J. J. Michalchuk, Y. Y. Jau, W. Happer, T. Mcclelland, H. Fruehauf, R. Drap, W. Weidermann, and M. Variakojis, "RF-interrogated end-state chip-scale atomic clock," in 39th Annual Precise Time and Time Interval Meeting, Long Beach, U.S.A., 27-29 November, 2007, pp. 233-248.

${ }^{33}$ M. Violetti, F. Merli, J. F. Zurcher, A. K. Skrivervik, M. Pellaton, C. Affolderbach, and G. Mileti, "New miniaturized microwave cavity for rubidium atomic clocks," in IEEE Sensors, Taipei, Taiwan, 28-31 October, 2012, pp. 1-4.

${ }^{34}$ V. Venkatraman, Y. Petremand, N. de Rooij, and H. Shea, J. Phys. D: Appl. Phys. 45, 505205 (2012). 